\title{
Diagnosis of brain death by transcranial Doppler sonography
}

\author{
H BODE, M SAUER, AND W PRINGSHEIM \\ University Children's Hospital, Freiburg, West Germany
}

SUMmARY The blood flow velocities in the basal cerebral arteries can be recorded at any age by transcranial Doppler sonography. We examined nine children with either initial or developing clinical signs of brain death. Soon after successful resuscitation increased diastolic flow velocities indicated a probable decrease in cerebrovascular resistance; this was of no particular prognostic importance. As soon as there was a clinical deterioration, there was a reduction in flow velocities with retrograde flow during early diastole, probably due to an increase in cerebrovascular resistance; this indicated a doubtful prognosis. In eight of the nine children with clinical signs of brain death a typical reverberating flow pattern was found, which was characterised by a counterbalancing short forward flow in systole and a short retrograde flow in early diastole. This indicated arrest of cerebral blood flow. One newborn showed normal systolic and end diastolic flow velocities in the basal cerebral arteries for two days despite clinical and electroencephalographic signs of brain death. Shunting of blood through the circle of Willis without effective cerebral perfusion may explain this phenomenon. No patient had the typical reverberating flow pattern without being clinically brain dead. Transcranial Doppler sonography is a reliable technique, which can be used at the bedside for the confirmation or the exclusion of brain death in children in addition to the clinical examination.

Only modern techniques of intensive care have made the diagnosis of 'brain death' possible. Brain death is the complete and irreversible cessation of brain function, even though the blood continues to circulate in the rest of the body. The death of the brain means the death of the person. ${ }^{2}$ Clinical criteria for brain death in adults and children have been established by various committees..$^{2-5}$ In addition, confirmation of brain death by laboratory tests is often necessary. Four vessel arteriography, ${ }^{6}$ radionuclide cerebral angiography, ${ }^{7}$ Doppler sonography of the extracranial brain supplying arteries, ${ }^{8}$ serial electroencephalograms, and brainstem auditory evoked potentials ${ }^{9}$ have also been used. These techniques have, however, inherent problems that limit their application.

With transcranial Doppler sonography it is possible to record the flow velocities in the intracranial basal cerebral arteries at any age. ${ }^{10}$ This technique is rapidly available, harmless, and reliable. ${ }^{10} 11$ The aim of our study was to find out if in children it is possible to confirm the clinical diagnosis of brain death by showing cessation of intracranial forward blood flow with transcranial Doppler sonography.

\section{Patients and methods}

Between September 1985 and December 1987, 27 unconscious children (Glasgow coma scale $<8$ ) were examined. Nine patients (eight of them after successful resuscitation) presented the clinical signs of either initial or developing brain death. These were deep coma, no spontaneous respiration, and no brain stem reflexes (pupillary, oculocephalic, corneal, pharyngeal, tracheal, or trigeminal pain reaction). Drugs, hypothermia, and endocrine or metabolic causes for coma had been excluded. The ages and clinical diagnoses are shown in the table.

Transcranial Doppler sonography was carried out by an EME TC 2-64 system (EME, Überlingen, West Germany) with a pulsed $2 \mathrm{MHz}$ Doppler probe and an integrated fast Fourier real time frequency analyser (sliding average technique, 64 frequency points, 132 spectral lines). The supraclinoidal segments of both internal carotid arteries were inson- 
Table Patients with clinical signs of brain death

\begin{tabular}{lll}
\hline $\begin{array}{l}\text { Case } \\
\text { No }\end{array}$ & Age & Diagnosis \\
\hline 1 & & \\
2 & 12 years & Near drowning \\
3 & 12 years & Severe head injury \\
4 & 10 years & Brainstem aneurysm \\
5 & 7 years & Mucoviscidosis \\
6 & 5 years & Severe head injury \\
7 & 16 months & Obstructive apnoea \\
8 & 12 months & Sudden infant death syndrome \\
9 & 3 months & Sudden infant death syndrome \\
\hline
\end{tabular}

ated by placing the Doppler probe on to the temporal bone between the lateral margin of the orbit and the ear, about $1 \mathrm{~cm}$ abvove the zygomatic arch. The ultrasonic beam was directed slightly forwards. ${ }^{10} 12$ In infants, these vessels were found with the probe on the anterior fontanelle with the beam directed towards the angle of the lower jaw. ${ }^{11}$ The basilar artery was insonated with the transducer in the midline of the neck and the ultrasonic beam directed through the foramen magnum. The measuring depth was adjusted to previously established age dependent values. ${ }^{11}$ Systolic peak flow velocity, mean flow velocity (time mean of the maximum velocity envelope curve), and early and late diastolic peak flow velocity were calculated, and compared with the age dependent reference values. ${ }^{11}$ Frequency to blood flow velocity conversions assumed an insonation angle of zero. In addition, the shape of the whole Doppler range during one cardiac cycle was analysed.

Arterial blood pressure was measured at the same time as the Doppler readings were taken. Carbon dioxide tension was measured either transcutaneously or from arterial blood samples.

Electroencephalograms were recorded with a Mingograf EEG 10 (Siemens, Erlangen, West Germany). Brainstem auditory evoked potentials were derived by a Tesy II device (Tönnies, Freiburg, West Germany). Real time cranial ultrasound through the anterior fontanelle was carried out with a Mark 100 (Advanced Technology Laboratories, Seattle, USA) with a short focused $5 \mathrm{MHz}$ transducer. Four vessel arteriography was done by catheterisation under fluoroscopic control.

The study was approved by the hospital ethical committee.

\section{Results}

During all Doppler readings of this series the arterial blood pressure was within the normal range for age, and the transcutaneous or arterial carbon dioxide tensions were above $3.33 \mathrm{kPa}$.

After successful resuscitation transcranial Doppler sonography showed normal systolic peak flow velocities and increased end diastolic peak flow velocities in the basal cerebral arteries (fig 1a). At the same time as the clinical deterioration occurred, but before the clinical signs of brain death were obvious, zero or even retrograde early diastolic peak flow velocities and anterograde end diastolic peak flow velocities were recorded (fig 1b). Finally, a characteristic reverberating flow pattern with a short and low forward flow in systole and a counterbalancing retrograde flow in early diastole was seen in both internal carotid arteries and in the basilar artery (fig 1c). In case 5 this pattern appeared in the internal carotid arteries some hours earlier than in the basilar artery. It was also recorded in cases 1-8. All patients died within 24 hours or on discontinuation

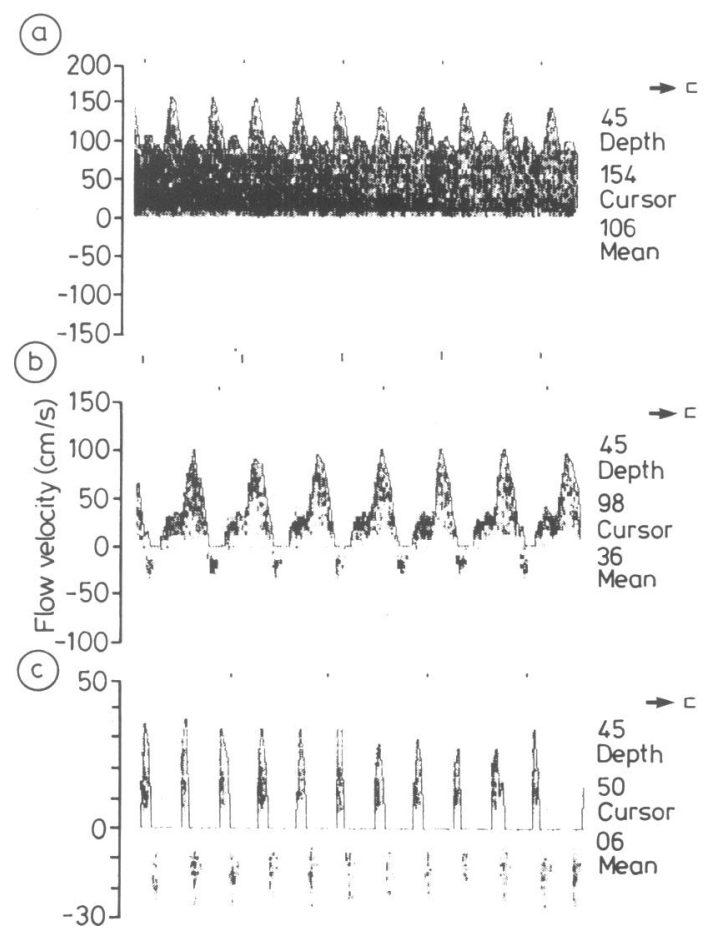

Fig 1 Progression to brain death. Transcranial Doppler sonography of left internal carotid artery. Depth = measuring depth in $\mathrm{mm}$, cursor=systolic peak flow velocity, and mean = time mean of the maximum velocity envelope curve. The arrow to the right indicates that a flow towards the probe is shown above the zero line. (a)-Three hours after resuscitation, (b)-24 hours after resuscitation, and (c)-with clinical signs of brain death. 
of mechanical ventilation. In case 9 a flow pattern as shown in fig 1a indicated the persistence of an intracranial circulation for two days, despite clinical and electroencephalographic evidence of brain death. Another infant presented with deep coma (Glasgow coma scale 5) and severe brain oedema shown on computed tomography after shaking trauma due to child abuse. At the same time as the clinical deterioration occurred, there was a reduction in the diastolic flow velocities in both internal carotid arteries, and an early diastolic retrograde flow was seen in the basilar artery (fig 2). The flow velocities increased again within 24 hours when there was a slight clinical improvement. This child survived in a vegetative state.

The electroencephalograms carried out in seven patients (cases 1, 2, 3, 4, 6, 7, and 9) showed isoelectric tracings either initially or later on. The intracranial components of brainstem auditory

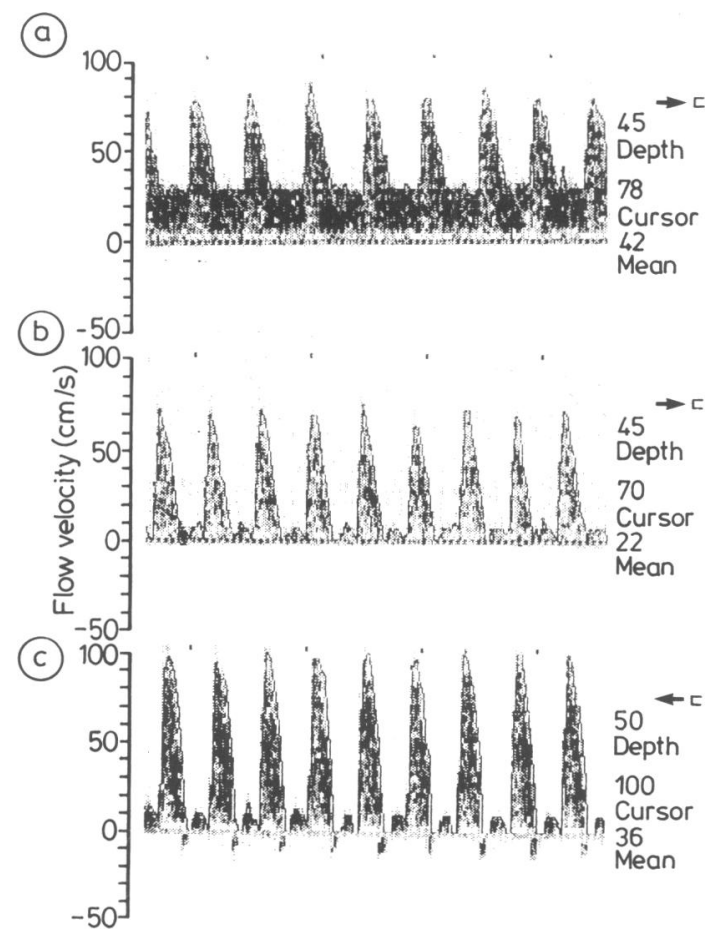

Fig 2 Cerebral shaking trauma-transcranial Doppler sonography. Depth=measuring depth in $\mathrm{mm}$, cursor $=$ systolic peak flow velocity, and mean = time mean of the maximum velocity curve. The arrow towards the left indicates that a flow away from the Doppler probe is shown above the zero line. (a)-Left internal carotid artery on day 4 after trauma, (b)-left internal carotid artery on day five, and (c)-basilar artery on day five. evoked potentials were derived in three patients. They were decreasing in case 1 and were already absent in the first recordings of cases 7 and 9. Real time cranial ultrasound was possible in three infants and showed diffusely increased echogenicity of the brain parenchyma. The pulsations of the intracranial arteries were absent in cases 7 and 8 , and in case 9 they persisted in the basal cerebral arteries.

Cerebral arteriography was carried out in case 3 after clinical, electroencephalographic, and Doppler sonographic confirmation of brain death before organs were removed for transplantation. There was no flow in either the two internal carotid or the vertebral arteries.

Examination at necropsy showed severe brain oedema (cases 2, 6, 7, and 8) with superior and inferior herniation (case 6), hypoxic/ischaemic neuronal damage (case 8), or liquification of the brain (cases 1 and 9).

\section{Discussion}

Though many authors accept the diagnosis of brain death by clinical criteria alone, ${ }^{345}$ additional laboratory tests are often used for confirmation or objective assessment. As these tests monitor either the circulation or the electrical activity of the brain their results are not always comparable. We will discuss only some tests of cerebral circulation.

Four vessel arteriography has shown arrest of cerebral blood flow and a characteristic to and fro movement of the blood below the base of the skull in the arteries supplying the brain. ${ }^{6}$ Because the injected contrast medium may aggravate preexisting brain oedema we use this technique only for confirmation and assessment before the removal of organs for transplantation when there is no doubt about the diagnosis of brain death. Radionuclide techniques are less invasive and give only small doses of radiation. They are less reliable, but can be used for diagnosing brain death. ${ }^{7}$ Doppler sonography has no known side effects and is not invasive. The Doppler technique records flow velocities in arteries supplying the brain, but cannot quantitatively determine cerebral blood flow. This, however, is not necessary for the diagnosis of brain death in which the cessation of intracranial forward blood flow alone has to be shown.

It has been shown that increased intracranial pressure may influence the Doppler velocity profiles in the intracranial arteries in infancy. ${ }^{13} 14$ In brain death different Doppler profiles have been recorded from different arteries. In adult brain death a to and fro movement and escape of carotid arterial blood externally was seen. ${ }^{8}$ In children with brain death 
there is a sharp systolic peak followed by a negative deflection, again a forward flow in early to mid diastole, then almost no flow at the end diastole in the common carotid artery. ${ }^{7}$ During the progression to brain death in neonates, there is a sequence of loss of diastolic flow, appearance of retrograde flow during diastole, diminution in systolic flow, and ultimately no detectable flow in the anterior cerebral artery despite considerable flow in the common carotid artery. ${ }^{5}$

Only transcranial Doppler sonography, which combines a low emitting frequency with the pulsed Doppler technique, can determine the intracranial blood flow velocities at any age. Characteristic reverberating Doppler profiles were recorded in the basal cerebral arteries of adults with brain death: brief systolic orthograde and early diastolic retrograde movements indicating net zero flow. ${ }^{12}$ In only a few adults the sound beam is unable to penetrate the skull, ${ }^{10-12}$ and then Doppler signals from the intracranial arteries cannot be recorded, and the diagnosis of brain death is impossible.

We present the first results of transcranial Doppler sonography in children with clinical signs of brain death initially or later on. The increased end diastolic flow velocities soon after resuscitation probably indicate the vasogenic phase of cerebral oedema that results from a decrease of the cerebrovascular resistance caused by vasodilation of the peripheral cerebral vessels. This is obviously a reversible phenomenon that we also saw in patients who had favourable outcomes.

We speculate that the prognostically doubtful pattern of reduced systolic flow velocities, retrograde flow velocities in early diastole, and then anterograde end distolic flow velocities, is caused by an increase of the cerebrovascular resistance. This could be a result of increasing brain oedema and the swelling of the endothelium of the small cerebral vessels. ${ }^{8}$ Changes in the compliance of the arteries supplying the brain may alter pulse wave reflexions from distally occluded arteries. The sharp Doppler signals with forward flow in systole and reversed flow in diastole are probably caused by the compliance of the basal cerebral arteries, and indicate the cessation of intracranial net forward blood flow in brain death. We never obtained the flow pattern shown in fig 1c in children who were not clinically brain dead. The 18 surviving children with temporary deep unconsciousness always showed anterograde pandiastolic flow; the flow pattern of fig $1 \mathrm{a}$ was often seen. The typical reverberating flow pattern in both internal carotid and the basilar artery is therefore highly specific for brain death, and we believe it can serve as a confirmatory test in children as well as in adults.
Our findings with transcranial Doppler sonography during progression towards brain death are similar to the results obtained from the common carotid artery in children who were brain dead. ${ }^{7}$ In contrast to that study, we found that an early diastolic retrograde flow in the basal cerebral arteries may indicate imminent brain death, but patients may also survive with severe deficits. In some cases, arrest of intracranial flow may not occur exactly at the same time in different basal cerebral arteries. It may also occur in severe infratentorial injuries. Under such circumstances clinical signs and brainstem evoked potentials may indicate brain death, while Doppler sonography still shows continuing blood flow in the arteries supplying the supratentorial parts of the brain. Further study of these problems is required.

On the other hand, in newborn infants flow velocities appropriate for age may persist through the basal cerebral arteries for some days in spite of brain death proved by clinical signs, electroencephalography, and necropsy. The flow velocities may not correspond to an appropriate cerebral blood flow because the diameter of the basal cerebral arteries is unknown. The flow may be decreased due to endothelial swelling, but the Doppler readings indicate a different pathophysiological process leading to brain death in this age group. We speculate that the blood is shunted through the circle of Willis and into extracranial arteries without cerebral perfusion. This phenomenon has been discovered by angiography in children who are brain dead. ${ }^{6}$ The persistent pulsations of the basal cerebral arteries alone in cranial ultrasound further support this. Therefore the diagnosis of brain death by Doppler sonography may be difficult or impossible in neonates. ${ }^{716}$ There are also problems of diagnosing brain death in neonates that are associated with the clinical signs, ${ }^{2}$ the electroencephalogram, ${ }^{9}$ and brainstem evoked potentials. ${ }^{17}$

The advantage of transcranial Doppler sonography for examining intracranial arteries in cases of suspected brain death when compared with Doppler techniques that only record the flow in the extracranial arteries supplying the brain is obvious; only transcranial Doppler sonography can record intracranial flow velocities at any age. Like other Doppler techniques it is dependent on the experience of the examiner, but the technique is comparatively easy to perform. It is not affected by artefacts or drugs that may make the evaluation of techniques that record the electrical activities of the brain on intensive care units difficult or even impossible. Finally, the rapid availability of the instrument and the speed with which the results are obtained makes it the preferred method for use at the bedside for 
excluding or confirming brain death in childhood after the clinical examination.

\section{References}

${ }^{1}$ Mollaret P, Goulon M. La coma depasse. Rev Neurol 1959;101: 3-15.

2 Stellungnahme des wissenschaftlichen Beirates der Bundesärztekammer. Kriterien des Hirntodes. Deutsches Ärzteblatt 1986;83: 2940-6.

3 Anonymous. An appraisal of the criteria of cercbral death: a summary. A collaborative study. JAMA 1977;237:982-6.

${ }^{4}$ Conference of Royal Colleges and Faculties of the United Kingdom: diagnosis of brain death. Lancet 1976;ii:1069-70.

5 Report of the medical consultants on the diagnosis of death for the President's commission for the study of ethical problems in medicine and biomedical and behavioral research: guidelines for the determination of death. JAMA 1981;246:2184-6.

6 Parvey LS, Gerald B. Arteriographic diagnosis of brain death in children. Pediatr Radiol 1976;4:79-82.

7 Ashwal S, Smith AJK, Torres F. Radionuclide bolus angiography: a technique for verification of brain death in infants and children. J Pediatr 1977;91:722-8.

8 Yoneda S, Nishamoto A, Nukada T, Kuriyama Y, Katsurada K, Abe $\mathrm{H}$. To-and-fro movement and external escape of carotid arterial blood in brain death cases: a Doppler ultrasonographic study. Stroke 1974;5:707-12.

${ }^{9}$ Lütschg J, Pfenninger J, Lundin H. Brainstem auditory evoked potentials and early somatosensory evoked potentials in neurointensively treated comatose children. Am J Dis Child 1983;137:421-6.

10 Aaslid R, Markwalder TM, Nornes H. Noninvasive transcranial Doppler ultrasound recordings of flow velocity in basal cerebral arteries. J Neurosurg 1982;57:769-74.

" Bode H, Wais U. Age dependence of flow velocities in basal cerebral arteries. Arch Dis Child 1988;63:606-11.

12 Harders A. Neurosurgical applications of transcranial Doppler sonography. Berlin: Springer Verlag, 1986.

13 Hill A, Volpe JJ. Decrease in pulsatile flow in the anterior cerebral arteries in infantile hydrocephalus. Pediatrics 1982; 69:4-7.

14 McMenamin JB, Volpe JJ. Doppler ultrasonography in the determination of neonatal brain death. Ann Neurol 1983;14: $302-7$.

is Ahmann PA, Carrigan TA, Carlton D, Wyly B, Schwartz JF. Brain death in children: characteristic common carotid arterial velocity patterns measured with pulsed Doppler ultrasound. J Pediatr 1987;110:723-8.

16 Altman DI, Perlman JM, Powers WJ. Exuberant brainstem blood flow and intact cerebral blood flow despite clinical and pathological evidence for brainstem and cerebral necrosis in an asphyxiated newborn infant. Ann Neurol 1986;20:409.

17 Dear PRF, Godfrey DJ. Neonatal auditory brainstem response cannot reliably diagnose brainstem death. Arch Dis Child 1985;60:17-9.

Correspondence to Dr H Bode, University Children's Hospital, Römergasse 8, CH 4005 Basel, Switzerland.

Accepted 6 June 1988 